\title{
Characterizing the zebrafish synaptic proteome
}

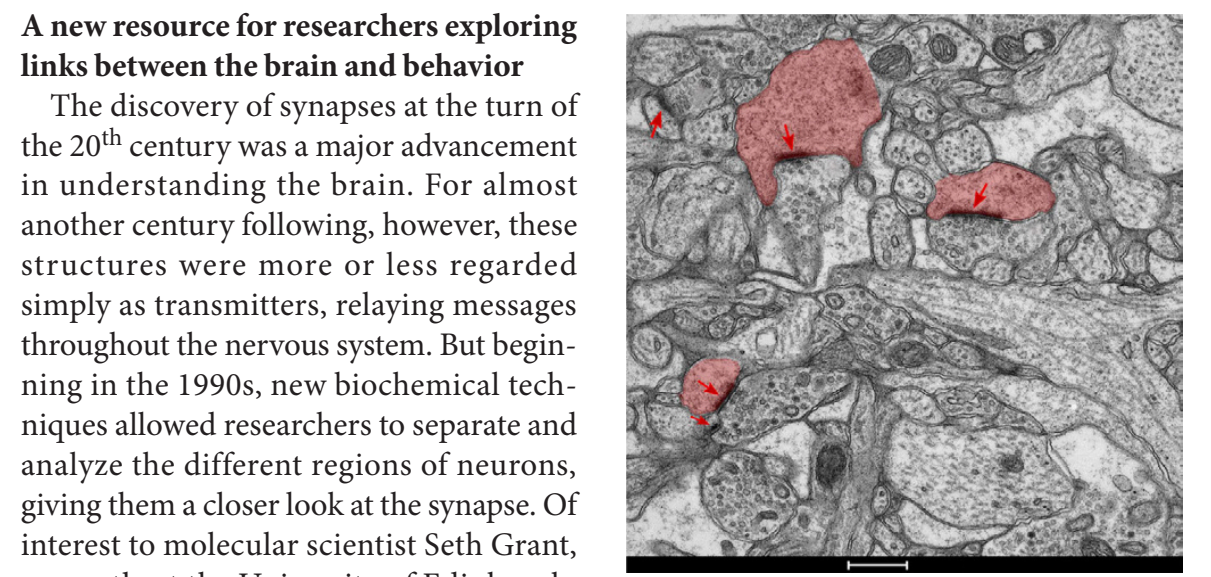
currently at the University of Edinburgh, was the synaptic proteome, the collection of proteins associated with synapses. Since their first proteomic paper published in 2000 , he and his colleagues have been systematically charting the complexity of the synaptic proteome in mice, humans, and even Drosophila; a new paper and its accompanying datasets add zebrafish to the series (Nat. Commun. 8, 14613; 2017).

"The synapse is in fact a super sophisticated molecular computational device," Grant explains, expressing over a thousand different proteins that receive, interpret, and relay signals from the brain. "And the postsynaptic density proteome is a kind of Rosetta Stone." The postsynaptic density, or PSD, is a particularly proteindense region of the receiving side of the synapse. Grant and his colleagues have documented previously that the behavioral repertoire of a given organism is related to the complexity of its PSD proteome; they have also demonstrated links between mutations in PSD proteins and over 130 different neurological diseases in humans. Understanding the synaptic proteome is thus an important component of cognitive and behavioral research.

Although the zebrafish is an increasingly popular neurological model, its synaptic
TEM image of zebrafish synapses. Adapted from Nat. Commun. 8, 14613; 2017.

proteome had never been characterized, nor, Grant and his colleagues found, had anyone imaged the structure of its synapses. The team first used transmission electron microscopes to create images of the ultrastructure of zebrafish synapses and confirm the presence of the PSD. In their images, they identified unique morphologies as well as some features that appear to be shared with mammals. They then created proteomic profiles from different fractions of zebrafish synapses, comparing those to concurrently prepared mouse samples and analyzing the resulting proteins and protein families against existing proteomic databases. This allowed them to compare and contrast proteomic results between zebrafish, mice, and humans.

The synaptic proteome of the zebrafish, it turns out, is more complicated than that of mammals-while paradoxically also being less so. Proteomic complexity in vertebrates is the result of two genome duplication events very early in their evolutionary history. About 150 million years after those vertebrate duplications, the teleosts-the bony fish clade containing zebrafish- experienced their own genome duplication event. Given that extra duplication, Grant expected straightforward results: the zebrafish synaptic proteome should theoretically contain more proteins that that of the mouse. It does, at least overall when considering the proteome of the entire synapse. But the PSD proteome, the subsection linked to behavioral complexity, actually contains fewer proteins. What that means for the computational power of the zebrafish brain warrants more investigation.

Regardless of the differences observed, comparing the datasets allowed the team to ask an important question: what's the same? Grant explains, "We can now identify about a thousand proteins in the fish, in the mouse, and in the human that are all highly conserved between them." He refers to these as the vertebrate postsynaptic density proteins, or vPSD, and anticipates that this set of molecules will turn up elsewhere in the vertebrate family tree. In addition to informing evolutionary history, knowledge of those homologues may help zebrafish researchers target their models for translational studies. For instance, if there is a known diseasecausing mutation in a human PSD gene that also exists in zebrafish, researchers could create transgenic fish with that specific genetic mutation to test potential treatments.

Though advising a little caution when designing studies involving zebrafish - to ensure their extra genomic duplication is accounted for-Grant hopes the dataset will prove useful to others interested in exploring the connection between synaptic complexity and behavior and disease. "With the capacity that there is with zebrafish to do large scale experiments," Grant says, "and so many different orthogonal datasets and statistical approaches that could be used, I expect to see some very interesting things coming from it."

Ellen P. Neff 